\title{
Vom Komitee zum Konzern - Das IOC und seine multimedialen Spiele
}

Holger Kühner

\section{Abstract}

Von den ersten Olympischen Spiele 1896 in Athen berichtete Pierre de Coubertin selbst. Als Generalsekretär und von 1896 an als Präsident des Internationalen Olympischen Komitees schrieb Coubertin für Zeitungen und Magazine. Auch IOC-Präsident Thomas Bach (seit 2013) ist als Berichterstatter tätig - meist auf den Social-Media-Kanälen des IOC, auf der eigenen Homepage oder im Olympic Channel. Zwischen Athen 1896 und Tokio 2020 liegt eine technische Evolution, die durch die Digitalisierung die nächste mediale Revolution erlebte. Anfangs ging es bei der Berichterstattung von den Olympischen Spielen nur um die sportlichen Wettbewerbe, die Leistungen der Olympiateilnehmer*innen und die kleinen Geschichten hinter den Kulissen. Im Sog der zunehmenden Kommerzialisierung entwickelte sich der Leistungssport $\mathrm{zu}$ einer spannenden und glamourösen Abteilung der Unterhaltungsindustrie. $\mathrm{Zu}$ den Film- und Popstars gesellten sich die Sportstars. Rolle und Anforderungen an Journalist*innen veränderten sich in einem ähnlich rasanten Tempo. Die glänzenden Medaillen offenbarten wie die Sieger*innen ihre Schattenseiten. Das „Gewinnen um jeden Preis“ wurde zum Nährboden für Doping und Korruption. Wurde 1964 das TV-Signal aus Tokio noch live über die USA und dann per Seekabel zurück nach Europa gesendet, wird die Übertragung heute über eine Cloud und weltweit vernetzte Glasfaserverbindungen abgewickelt. Mitbeteiligt an den technischen Innovationen sind häufig auch die TOP Sponsoren des IOC, die nicht nur Geld bezahlen, sondern auch Sachleistungen zur Verfügung stellen sowie speziell auf die Bedürfnisse der Olympischen Spiele zugeschnittene Produkte entwickeln. Mit der Gründung der Olympic Broadcasting Services (OBS) 2001 begann das IOC, die TV-Bilder der Olympischen Spiele selbst zu produzieren. Mit dem Olympic Channel, der digitalen Plattform für Olympische Inhalte schuf das IOC 2016 seinen eigenen „TVSender" und wurde damit zu einer News Producing Company (NPC). In 125 Jahren seit den ersten Spielen von Athen 1896 hat sich das Internatio- 
nale Olympische Komitee in einen Konzern verwandelt. Durch die Attraktivität der Olympischen TV-Rechte rutschte das IOC aber auch in eine finanzielle Abhängigkeit - und mit ihm der gesamte Olympische Sport, der auch von den IOC-Einnahmen lebt. In der Finanzperiode 2013-2016 kamen $73 \%$ aller Erlöse des IOC von TV-Unternehmen, die dafür bezahlen, exklusiv von den Olympischen Spielen berichten zu dürfen.

\section{Olympische Spiele und die Medien - eine Beziehungsgeschichte}

„Durch die Beschleunigung der Digitalisierung werden wir mit Sicherheit eine nie dagewesene Anzahl von Fans und Menschen haben, die die Spiele im Internet und an den Fernsehschirmen verfolgen“, sagte IOC-Präsident Dr. Thomas Bach (Bach, 2020) mit Blick auf die Olympischen Spiele in Tokio (23.07.- 12.08.2021). Nachdem die japanische Regierung drei Wochen vor Beginn der Spiele erneut den Notstand ausrief, entschieden die Olympia-Organisatoren: die Olympischen Spiele in Tokio finden ohne Zuschauer statt. Dafür mit medialen Rekorden - im Fernsehen, im Internet, am Tablet und Smartphone: „Das wird nach allem, was wir bisher sehen, alles bisher Dagewesene übertreffen, so dass zumindest in der digitalen Welt und der Fernsehwelt dieser Enthusiasmus da sein wird“ (Bach, 2020). Sechzig Jahre nachdem in Rom erstmals TV-Rechte verkauft wurden, hat sich aus dem Internationalen Olympischen Komitee der Amateure ein internationaler Medienkonzern entwickelt. Die Machtverhältnisse sind eindeutig. Das IOC vermarktet die Olympischen Spiele und verteilt die Einnahmen. Alle, die aus der Olympischen Familie mitspielen wollen, verdienen mit - ihre finanzielle Abhängigkeit vom IOC ist enorm. Mit der Gründung der IOC eigenen Produktionsfirma Olympic Broadcasting Services (OBS) sorgt das IOC auch für journalistische Inhalte überall dort, wo bewegte Bilder laufen können: im klassisch linearen Fernsehen, im Internet und auf Social-Media-Plattformen. Mit dem Olympic Channel, der seit 2016 sendet, schuf das IOC einen eigenen "Sender“. Das größte Sportereignis der Welt dient alle vier Jahre auch als „technisches Experimentierfeld“. Die TV-Rechteinhaber (Rightsholding Broadcaster - RhB) bezahlen also nicht nur für die exklusiven Übertragungsrechte, sie profitieren auch von den Investitionen und Innovationen neuer Produktionsund Übertragungstechniken. Was als Prototyp bei Olympischen Spielen getestet wird, können die Sender nach den Olympischen Spielen für die tägliche Berichterstattung nutzen, weiterentwickeln oder einfach übernehmen. Heute kommen $73 \%$ aller Einnahmen des IOC aus dem Verkauf von Übertragungsrechten (IOC, 2020a). Diese Einnahmequelle 
bringt dem IOC finanzielle Stabilität, führt aber auch zur Abhängigkeit von diesen Einnahmen. Der amerikanische Medienkonzern NBCUniversal (NBCU) sicherte sich bereits im Mai 2014 die Rechte für die USA bis ins Jahr 2032 - für 7,65 Milliarden USD (IOC, 2014a). Was der NBCU-Deal tatsächlich wert ist, lässt sich daher schwer vorhersagen. Wer immer höhere Preise für Übertragungsrechte fordert, muss aber auch die Gegenleistung immer attraktiver gestalten. Der technische Fortschritt in der digitalen Welt vollzieht sich in Höchstgeschwindigkeit. Entwicklungszyklen neuer Medienprodukte beginnen meist mit einer Testphase bei Winterspielen, um sie dann zwei Jahre später bei Sommerspielen den RhBs voll einsatzfähig anbieten zu können. Für die weltweite Übertragung der Olympischen Spiele in Tokio entwickelte OBS mit dem chinesischen IT-Giganten und IOC-Partner Alibaba eine innovative Cloud-Lösung. Für die besten Athlet"innen nur die beste Technik. Sagte IOC-Präsident Avery Brundage bei den Olympischen Spielen 1972 beinahe beschwörend „the Games must go on", liest sich das im digitalen Zeitalter auf der gemeinsamen Homepage von Alibaba und OBS so: „keeping the Games uninterrupted“ (Alibaba \& OBS, 2021). Die Spiele finden nicht mehr nur in Rio, Sotschi und Paris statt, sondern immer und überall.

Olympische Geschichte der Olympischen Geschichten - von der Zeitung in die Cloud

„Die Olympischen Spiele werden hauptsächlich fürs Fernsehen inszeniert." Jacques Rogge $e^{1}$

Schnell und kompakt muss es zugehen, wenn Medien an der olympischen Berichterstattung teilnehmen. Weil einem Reporter des „Le Petit Havre“ bei seiner Berichterstattung über den Olympischen Kongress 1897 die Bezeichnung „Internationales Komitee für die Olympischen Spiele“ offenbar zu sperrig war, verkürzte er die Organisation auf „Internationales Olympisches Komitee“ (Samaranch 1996, S.9), die seit 1901 offizielle Bezeichnung. Schreibende Journalisten und Fotografen machten den Anfang. Die Erfindung der Cinematografie brachte erste Bewegtbilder in die Wochenschauen der Kinos. Bevor das Fernsehen zum visuellen Massenmedium wurde, dominierte die Radioberichterstattung. Aber kein

1 Barney, Wenn, Martyn (2002, S. 278). 
anderes Medium hatte einen vergleichbaren Einfluss auf die Entwicklung der Olympischen Spiele wie das Fernsehen. Verbände änderten Regeln und Formate ihrer Sportarten, um sie „telegen“ zu machen. Und aus reinen Fernsehanstalten, die anfangs nur zur Übertragung von Bewegtbildern zuständig waren, entwickelten sich Medienunternehmen, die olympische Inhalte über Multi-TV- und Digitalplattformen senden und verbreiten. Mit der Gründung des Olympic Channel 2016 wurde das IOC selbst zu einer "News Producing Company“, die journalistische Inhalte selbst herstellt, distribuiert und verkauft. Ein reines Sportereignis waren die Spiele aber noch nie, sondern aus Sicht der Medien auch ein Labor, in dem neue Technologien getestet und weiterentwickelt werden.

Den 100-Meter-Lauf bei Olympischen Spielen im Fernsehen oder im Radio zu erleben, erzeugt Spannung, Emotionen und ein Miterleben, das beim Lesen eines Zeitungsartikels am Tag danach kaum entstehen kann. Deshalb hat Downing (1996, S. 27) recht, wenn er die Berichterstattung von den Olympischen Spielen 1936 in Berlin als Durchbruch sieht: „Berlin markiert einen zentralen Meilenstein für den Einfluss, den die Medien zur Entwicklung der Olympischen Spiele haben und begründete die neue Ära des Fernsehens". Die von den Nationalsozialisten für Propagandazwecke benutzten Spiele wurden im Radio in 40 Länder übertragen. In der Dokumentation „Olympia-Weltsender" wird darauf hingewiesen, dass beispielsweise NBC-Reporter aus Berlin allein für 110 Radio-Programme in den USA berichteten (Reichs-Rundfunk 1936). In einem Radius von 15 Kilometern wurden die Spiele live im Fernsehen gesendet und nach 90sekündiger Entwicklungszeit der Filme als Videosignal in öffentliche Zuschauerräume übertragen (Llinés \& Moreo, 1998).

Bereits drei Jahre nach Ende des 2. Weltkriegs wurden 1948 in London Filme hergestellt. Bei den Schwimmwettbewerben wurden Sportler noch "schnaufend und triefnass" interviewt (Hampton, 2008, S. 47). Die Übertragungen waren so erfolgreich, dass die BBC ihr Programm ändert und die Olympischen Spiele zeigte, insgesamt 70 Stunden an 15 Tagen. Nach Downing (1996, S. 28) bleibt das Fernsehen noch der Juniorpartner der Kinos, dennoch mache die Berichterstattung den „ersten Schritt hin zum kleinen Monitor“. Es dauerte allerdings bis zu den Spielen 1956, bis das Fernsehen auch von den Olympiamachern im IOC als Transportmittel von Informationen und Emotionen entdeckt wurde.

Obwohl dem IOC die Olympischen Spiele gehören, verkaufte es sein Produkt nicht selbst an TV-Sender. Als bei der Eröffnungsfeier der Olympischen Winterspiele 1956 in Cortina d'Ampezzo der letzte Fackelläufer über ein Mikrofon stolperte, soll Avery Brundage in einer anschließenden IOC-Sitzung gesagt haben: „Das IOC ist 60 Jahre ohne das 
Fernsehen ausgekommen und wird es auch in den nächsten 60 Jahren“ (Lyberg, 2004). Doch diese Aussage hatte nur ein halbes Jahr Bestand. Bei der Exekutivkomiteesitzung im Oktober 1956 in Lausanne verwies Brundage (IOC, 1956a) darauf, dass amerikanische Firmen bereit seien, für TV-Rechte bei wichtigen Sportevents „astronomische Preise“ zu bezahlen.

Erstmals tauchte der Verkauf von TV-Rechten in der Olympischen Charta 1958 auf. Die Fernsehrechte zur Live-Übertragung von den Spielen sollten vom Organisationskomitee unter der Aufsicht des IOC (IOC, 1958) verhandelt und verkauft werden, doch da waren die Organisationsverträge für die Olympischen Spiele 1960 und 1964 schon geschlossen. Das IOC musste nicht nur zuschauen, wie die Organisationskomitees Verträge mit TV-Sendern schlossen und die Einnahmen für sich behalten wollen, es begann damit auch eine Zeit, die geprägt war von Konflikten zwischen dem IOC, den OKs und den Internationalen Verbänden. Dabei ging es immer um die Verteilung der Gelder. Die Einnahmen standen im Konflikt mit dem Amateurparagrafen des IOC. Das Image der Spiele zu schützen, war ein idealistisches Unterfangen, das Professionalisierung und Kommerzialisierung ablehnen musste. Im IOC glaubte man, dass es hilfreich sei, wenn die OKs die TV-Rechte verkauften, um das IOC aus kommerziellen Konflikten rauszuhalten. Immerhin mussten die OKs auch die Produktions- und Infrastrukturkosten bezahlen. Für das IOC selbst, sowie für die Internationalen Verbände war zunächst wenig zu holen. 


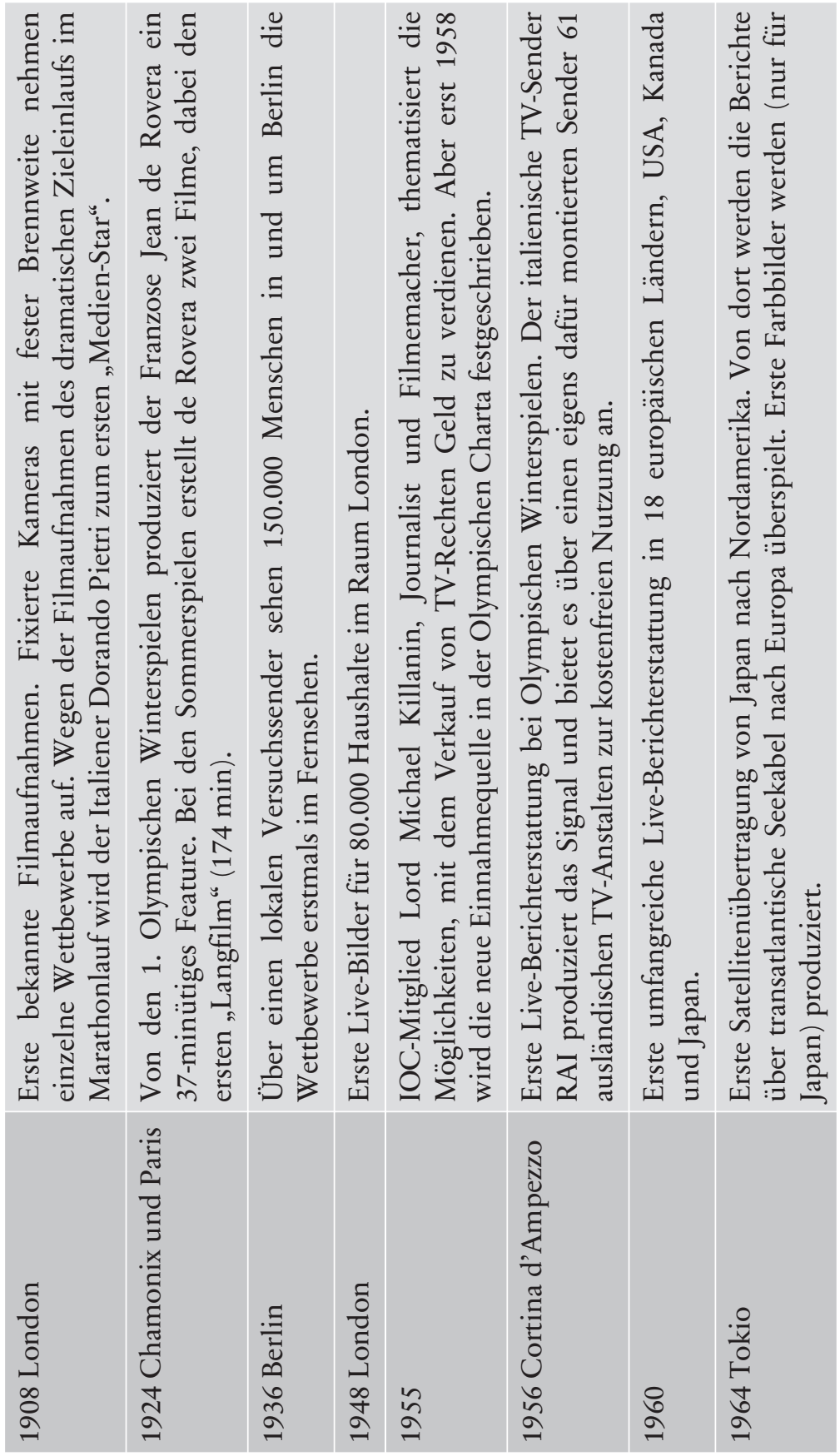




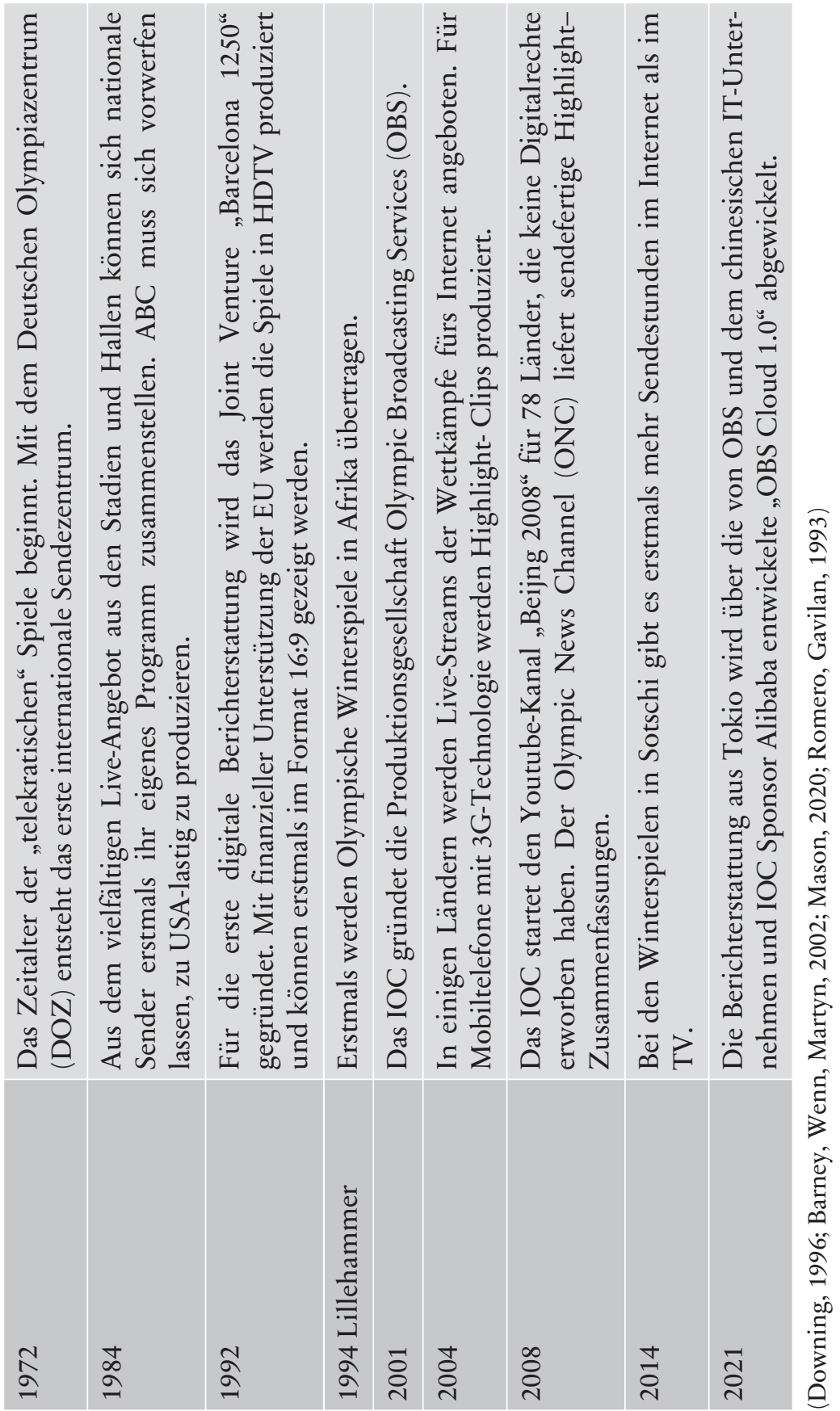


Erst für die Spiele 1972 wurde eine Lösung gefunden. Die „Rom Formel“, beschlossen bei der IOC-Session 1966 (IOC-Vollversammlung) in der italienischen Hauptstadt - kurz bevor die Sommerspiele an München und die Winterspiele an Sapporo vergeben wurden. Von der ersten Million aus dem Verkauf der TV-Rechte profitierten das IOC, die Internationalen Sportverbände (IF) und die Nationalen Olympischen Komitees (NOC) zu je einem Drittel. Die Organisationskomitees (OK) bekamen von der ersten Million nichts. Spannend wurde es für Organisatoren der Spiele, die immer noch die TV-Rechte verhandelten und verkauften, von der zweiten Million an. Die OKs bekamen 2/3 der TV-Einnahmen, IOC, IFs und NOKs je 1/9. Je besser die OKs verhandelten, desto mehr Geld floss. Doch die Erwartungen an die neue Geldquelle erfüllte sich auch in München noch nicht, „die Einnahmen rührten im Wesentlichen aus einem einzigen Vertrag her, dem Vertrag mit der American Broadcasting Company (Deutscher Bundestag, 1975, S.33). In München zählte viel mehr „der erhebliche ideelle Gewinn, den die Fernsehübertragungen in aller Welt für die Meinungsbildung über das Gastgeberland erbracht haben“. Die Abgrenzung zu den Nazi-Spielen 1936 in Berlin gelang, die Formel „36 $+36 \neq 72^{“}$ ging auf, auch wenn der SPIEGEL am 28. August 1972 titelte: „Olympia - das totale Fernsehen“. Vier Jahre vor den Spielen hatten ARD und ZDF am 21. Mai 1968 das „Deutsche Olympia Hörfunkund Fernsehzentrum" (DOZ) gegründet. Es diente als Vorbild für spätere Host-Broadcaster. Als Geschäftsführer wurde der Fernsehmoderator und stellvertretende ARD-Koordinator Robert Lemke („Was bin ich?“) eingesetzt (DOZ, 1974). Im Olympiapark entstand ein moderner Sendekomplex mit Fernsehstudios, Schnitt- und Redaktionsräumen. Der Zeitplan, den das DOZ mit dem OK und den Internationalen Verbänden ausarbeitete, war ein Kompromiss. Wer für die Übertragungen bezahlte, der wollte auch mitbestimmen. Die Forderungen der 70 TV-Organisationen nach attraktiven Wettkampfzeiten führten zu „kuriosen Startzeiten“ (DOZ, 1974). Handball- und Basketballfinale begannen erst um 23:00 Uhr. Im Abschlussbericht des DOZ (1974) heißt es: „Dabei zeigte es sich jedoch, dass viele der Funktionäre unmodern über den Umgang mit den modernen Medien Fernsehen und Radio dachten." Unterwasserkameras, eine mobile Kamera beim Marathon und ein Hubschrauber, der als fliegende Relais-Station diente, dazu die ARD Olympia-Welle im Radio. ARD Olympia-Teamchef Rudi Michel schrieb: „Die Qualität der vom DOZ angebotenen Bilder (Elektronik) ist unbestritten“ (Michel, 1972). München setzte neue TV-Maßstäbe. Die modernen Kamera- und Übertragungsmöglichkeiten hatten aber auch fatale Folgen. Das Fernsehen berichtete live über die Geiselnahme im Olympischen Dorf. Die Attentäter 
schauten mit, erfuhren so, dass die Polizei die Erstürmung der besetzten Wohnung plante.

Bis 1960 waren die Nutzer vor allem auf journalistische Texte in Tageszeitungen und Zeitschriften angewiesen. Sehen konnte man die Spiele in den Wochenschauen der Kinos, da kannte man die Ergebnisse aber meist schon aus der Zeitung. Wirklich dabei sein konnten die Menschen erst, als das Fernsehen den Sport in die Wohnzimmer brachte. Sie konnten mit den Sportlerinnen und Sportlern leiden, sie anfeuern und mit ihnen feiern. Die Zuschauermassen waren im Medium Fernsehen angekommen und mit ihnen das größte Sportereignis der Welt. Auch im 21. Jahrhundert heißen Überspielungen - ob live oder aufgezeichnete Stories - in der TVSprache „feed“ (Futter). Davon können vor allem die zahlungskräftigsten und damit einflussreichsten Rechteinhaber aus den USA nie genug haben. Schon 1972 erkannte Regiekoordinator Uly Wolters: „Immer nur Knall, Schuss, bumms, raus, weg - das wäre ja schrecklich“ (SPIEGEL, 1972, S. 29).

Verschiebung der Macht - das IOC wird "News Producing Company“

„Ohne das Fernsehen würden die Olympischen Spiele in einer Nische verschwinden."

Thomas Bach ${ }^{2}$

Die Diskussionen um die Verhandlungspositionen und die Verteilung der TV-Gelder an die Mitglieder der Olympischen Spielen hatten sich seit den 1960er Jahren zu einem Dauerkonflikt entwickelt. Kaum waren die Spiele an eine Stadt vergeben, hatte das IOC nur noch wenig Einfluss auf die Organisation und die Vorbereitungen der TV-Berichterstattung. In Montreal 1976 musste das OK mehr Geld für den Aufbau der technisch aufwändigen Medieninfrastruktur ausgeben, als es über den Verkauf der TV-Rechte einnahm. In seinen Erinnerungen „My Olympic Years“ schrieb der ehemalige IOC-Präsident Lord Michael Killanin: „Dieser eine spezielle Bereich, in dem das IOC immer einen oder zwei Schritte hinterher zu sein schien, war das Fernsehen. Niemand begriff schnell genug den Wert und die Gefahr, die von diesem Medium für die Entwicklung der Spiele ausging"(Killanin, 1983, S. 77).

$2 \operatorname{IOC}(2019 b)$. 
Das änderte sich erst als der Spanier Juan Antonio Samaranch 1980 zum Präsidenten gewählt wurde. Die beiden Boykottspiele in Moskau und Los Angeles veränderten die Ausrichtung der Olympischen Spiele. „Games over" war realistischer als die Botschaft „The Games must go on", die der damalige IOC Präsident Brundage bei der Trauerfeier nach dem Terroranschlag 1972 in München ausrief. Los Angeles, einziger Bewerber für 1984, organisierte die ersten privatwirtschaftlich finanzierten Spiele. Neben dem IOC, den NOKs, den IFs und den Athlet"innen kamen neue „Player“ in Spiel. Sponsoren, die bereit waren, viel Geld dafür zu bezahlen, um dabei sein zu dürfen. Bereits 1981 hatte Samaranch die Arbeitsgruppe „New Sources of Finance" eingerichtet. Das Ziel: Die Abhängigkeit von den TV-Einnahmen reduzieren, neue Finanzquellen schaffen und sich somit gegen ökonomische und politische Einflüsse absichern (IOC, 2014b).

Im Jahr 1982 gründete Adidas-Chef Horst Dassler die Vermarktungsagentur International Sports Leisure (ISL) und verkaufte im Auftrag des IOC Sponsorenpakete. Dassler legte "The Olympic Programme“ auf kurz: TOP. Die Anzahl der Firmen wurde limitiert, was die werbliche Attraktivität der Spiele hervorheben sollte. Für einen zweistelligen Millionenbetrag durften sich Firmen mit der sperrigen Bezeichnung „The Olympic Programme"-Sponsor schmücken oder einfach, schlagkräftig und werbewirksam abgekürzt: TOP-Sponsor. Nur die Besten schaffen es zu den Olympischen Spielen, die Top-Athlet*innen und die TOP-Sponsoren. Allein der Name ist bis heute ein gelungener PR-Schachzug. Die Kommerzialisierung wirkte wie eine Zentrifugalkraft, der Kreis drehte sich immer schneller. Heute versammeln sich unter „The Olympic Partners (TOP) Programme“ 15 internationale Sponsoren.

Über Veränderungen und Ergänzungen in der Olympischen Charta (Adi, 2013) und in den Host-City-Verträgen übernahm das IOC Schritt für Schritt die alleinige Verhandlung der TV-Rechte und die Verteilung der Einnahmen. Nach Los Angeles 1984 begann das, was Pound (2004) als „Broadcast Bonanza“ bezeichnete. Von Samaranch 1983 zum Vorsitzenden des „IOC Television Negotiations Committee“ bestimmt, begann IOCMitglied Richard Pound für die beiden Spiele 1988 in Calgary und Seoul ein Pokerspiel um die TV-Rechte, hauptsächlich auf dem US-Markt. In den Ausrichterverträgen für Albertville und Barcelona 1992 wurde das IOC erstmals als Verhandlungsführer festgeschrieben. Gespräche sollten „in Absprache mit den Organisationskomitees" geführt werden (Pound, 2004, S. 171). Damit hatte das IOC die Kontrolle über die wichtigste Einnahmequelle übernommen und die Machtverhältnisse nachhaltig verschoben.

Rekorde finden sich nun nicht mehr nur auf Siegerlisten, sondern auch in TV-Verträgen. Am 26. Januar 1993 schrieb die Deutsche Presse Agentur, 
dpa: „Olympischer Rekord: EBU zahlt 250 Millionen Dollar für Atlanta“ (dpa, 1993). Während sich der TV-Markt immer rasanter entwickelte, die Rechtekosten und Übertragungszeiten stiegen, stand das IOC aber immer noch vor einem doppelten Dilemma: Es hatte keinen Einfluss auf die Herstellung der TV-Bilder. Zudem dauern Olympische Spiele nur knapp drei Wochen und das kostbare Filmmaterial verschwindet danach wieder in den Archiven.

Zwei Jahre nach den Spielen in Barcelona sprach IOC-Mitglied Thomas Bach beim Olympischen Kongress in Paris erstmals davon, dass das IOC seine Bilder selbst produzieren sollte. Das sensible Dreiecksverhältnis zwischen Sport, Ökonomie und Fernsehen werde sich durch digitale Technologien verändern. Vor allem bei den Entwicklungen im TV stecke die Gefahr, dass der Sport nicht mehr sei als „fast food entertainment“ (Bach, 1994, S. 160). Das IOC müsse die Nutzung der TV-Rechte stärker überwachen, sagte Bach (1994, S. 160): „Die Produktion von Fernsehbildern, sowie die Übertragungen unter der alleinigen Kontrolle des IOC könnte den TV-Sendern helfen, den Sponsoren und vor allem dem Sport und der Olympischen Bewegung." Damit das IOC seine Olympischen Werte weiterhin lukrativ vermarkten konnte, musste es die Bilder und Botschaften dazu künftig selbst produzieren und distribuieren. Parallel arbeitete das IOC daran, Daten vergangener Spiele nicht nur zu sammeln, sondern verlässlich aufzubereiten und den Berichterstattern zur Verfügung zu stellen. Aus dem „Olympic Data Feed“ werden heute animierte Infografiken für Broadcast und Netcast hergestellt und deren Zuschauer informiert. Ende 1995 ging die erste Homepage des IOC online (Moragas, 1999). Die ersten „Internet Games“ (IOC, 1996) in Atlanta boten noch keine Videos, sondern ausschließlich Daten und Informationen. TVKommentatoren konnten das Commentator Information System (CIS) verwenden, Internet-Nutzer das Info'96 System über „www.atlanta.olympi c.org“ - ein Link, der heute direkt auf die IOC-Homepage führt.

In Sydney 2000 sahen manche das Internet schon als den stillen Sieger im Ringen um Aufmerksamkeit. NBC ging mit einer eigenen Homepage (NBCOlympics.com) an den Start, betrieben vom Internet-StartUp-Unternehmen Quokka aus San Francisco (Simon, 2000). Quokka experimentierte bereits mit animierten Grafiken und 360-Grad-Videos, die aus Telemetrie-Daten der sportlichen Bewegungen zusammengesetzt wurden. Im Gespräch mit Maney (2000) sagte Quokka-CEO Alan Ramadan, „die Olympischen Spiele sind für uns das, was Schneewittchen für Disney war" - ein Volltreffer. NBC sieht das eigene Engagement im Netz damals noch kritisch und als Konkurrenz zur Berichterstattung im Fernsehen. Im Silicon Valley geht es weniger um Journalismus, was zählt ist das Business. 
Im August 2000 übernimmt NBC 22 Prozent Anteile von Quokka - für 140 Millionen US-Dollar (CNNMoney, 2000).

Mit der Gründung des hundertprozentigen Tochterunternehmens Olympic Broadcasting Services (OBS), bereitete das IOC 2001 die Übernahme des TV- und Digitalgeschäfts vor. Zwei Jahre später wurde OBS in Madrid registriert. CEO wurde der Spanier Manolo Romero, der seit 1992 (meist mit seiner Firma International Sports Broadcasting) als Host Broadcaster beteiligt war. Weil die Verträge mit künftigen Ausrichterstädten bereits geschlossen waren, konnte OBS erst 2010 in Vancouver als alleiniger Host Broadcaster auftreten (Mason, 2020). Parallel nahm die Entwicklung der Digitalisierung Fahrt auf. Medienunternehmen begannen Sportwettbewerbe nicht nur im Fernsehen, sondern über Internet-Plattformen zu streamen. Nach dem Motto „all you can send“ treibt OBS die Übertragungen von Olympischen Spielen zu immer neuen Rekorden, wobei die TV-Sender kaum hinterherkamen.

Den nächsten Schritt zur kompletten Übernahme der TV-Kontrolle machten IOC und OBS in Peking. Der Youtube-Kanal „Beijing 2008“ des IOC war in Ländern empfangbar, für die keine Internetrechte verkauft waren. Somit wurde das IOC selbst zum Broadcaster. Fertig produziert wurden zudem 30-minütige Zusammenfassungen für den „Olympic News Channel“ (ONC). Wer es sich nicht leisten konnte, Reporter nach Peking zu schicken, übernahm die sendefertigen Produkte. In Vancouver 2010 wurde der ONC als 24-Stunden-Programm angeboten und kann als Vorläufer des „Olympic Channel“ gesehen werden.

\section{Der Olympic Channel}

Der Olympic Channel ist die digitale Plattform des Internationalen Olympischen Komitees. Der Kanal sendet seit 2016 digital - erreichbar über olympics. com, „Olympic Channel“-App, Social Media und in einigen Ländern auf TV-Kanälen. Angeboten werden exklusiv produzierte „,behind-the-scenes“ Filme, Serien, Interviews, sowie Live-Übertragungen. Das „over-the-top“ Angebot (OTT) ist kostenlos und frei zugänglich. Wie Netflix, Amazon Prime oder DAZN kommt der Olympic Channel ohne Provider aus. Nutzer können sich ihr Olympisches Programm 24/7 selbst zusammenstellen.

Thomas Bach packte das Projekt als eine Art Mitgift in seine Bewerbungsschrift zur IOC-Präsidentschaft. Bach (2013) empfahl, eine größere TVPräsenz auch in der Zeit zwischen den Olympischen Spielen herzustellen und einen „Olympic TV Channel“ zu schaffen. Im Jahr 2014, Bach ist nun IOC-Präsident, werden 40 Reformvorschläge der IOC Agenda 2020 
beschlossen. Unter der Überschrift „Olympism in action: keep Olympism alive 365 days a year" wird nur ein Thema aufgeführt und beschlossen: "Start eines Olympic Channel“ (IOC 2014c, S. 19). Der Channel richtet sich vor allem an die jugendliche Zielgruppe. Die Olympischen Spiele „to go" und „24/7" auf dem Tablet und Smartphone. Man muss nicht mehr zum Fernsehgerät gehen und warten bis die Übertragungen beginnen, man entscheidet selbst, wann und was man schaut. Noch in Rio de Janeiro geht der Olympic Channel auf Sender und füllt die Lücke zwischen den Olympischen Spielen. Die Dauerwerbesendung „Olympia“ beginnt.

Seit das IOC über OBS die Bilder selbst produziert, hat es die Macht über die Bilder und die Inhalte. Wer die TV-Bilder herstellt, mit denen Milliarden Menschen erreicht werden, kann seine Botschaften durchsetzen. Mit dem Olympic Channel erreicht das IOC Menschen in fast jedem Winkel der Erde. Zusätzlich sorgen TV-Unternehmen, die Übertragungsrechte an den Olympischen Spielen kaufen, für die massenhafte Verbreitung von Bildern und Botschaften und erfüllen darüber hinaus für das IOC weitere Zwecke. Sie bezahlen sehr viel Geld, sichern 73 Prozent der gesamten Einnahmen (IOC, 2020a). Sie kennen die landesspezifischen Bedürfnisse der Olympia-Fans, promoten die Olympische Bewegung. Sie setzen neue Technologien ein, um immer mehr Menschen über ihre Multi-TV- und Digital-Plattformen zu erreichen. Die TV-Sender nutzen die Olympischen Spiele ihrerseits, um maximale Reichweiten zu erzielen. Sie profitieren dabei auch von Innovationen, die das IOC/OBS mit seinen Wirtschaftspartnern der IT- und Technologiebranche entwickelt.

Heute ist das IOC nicht mehr nur eine Organisation, die mit den Olympischen Spielen die größte Sportveranstaltung der Welt organisiert. Das IOC hat sich zu einem internationalen „in-house“- Medienunternehmen entwickelt, das Fernsehbilder und Inhalte für multimediale Plattformen selbst produziert, distribuiert und verkauft. Das IOC kann daher auch als „News Producing Company“ (NPC) bezeichnet werden. Mit dem Internet und den Social-Media-Plattformen verändert sich auch das journalistische Angebot. Nachrichten werden nicht mehr nur über „Presse, Funk und Fernsehen" verteilt. Verbände, Eventveranstalter und vor allem Athlet*innen können Nachrichten nun selbst publizieren. Aus den Informationsquellen dieser neuen NPCs sprudeln Unmengen von Nachrichten, die kaum noch gefiltert werden können. Das Mediennutzungsverhalten ändert sich. Moragas (1999) identifiziert bereits vor der Jahrtausendwende eine entscheidende Herausforderung. Es sei absurd, anzunehmen, dass User allein durch Surfen und Suchen direkt die für sie richtigen Informationen finden würden. Dieser Trugschluss würde einen Aspekt übersehen: die notwendige Produktion von speziellen Inhalten. Zwanzig Jahre später 
ist die www-Welt nicht genug. Bei den Olympischen Spielen in Tokio wurde die Berichterstattung über eine Cloud (Wolke) gesendet. Bei der Vorstellung der „OBS Cloud 1.0“ sagte Yiannis Exarchos, CEO von OBS: „Als Produzenten der Übertragung der Olympischen Spiele sehen wir uns bei OBS als Vorreiter bei der Umgestaltung der Medienbranche (IOC, 2018).

Olympische Spiele im digitalen Zeitalter - ein Geschäft auf Gegenseitigkeit

Das IOC selbst publiziert immer neue Rekorde zu den weltweiten Sendezeiten, Reichweiten und Einnahmen durch den Verkauf von TV-Rechten. Bei den Olympischen Spielen in Rio de Janeiro konnten wieder einige Rekorde gebrochen werden. Die mit exklusiven Übertragungsrechten gesegneten TV-Sender berichteten - alle zusammen - 365.924 Stunden aus und über die Spiele, im Fernsehen und auf ihren digitalen Plattformen. Erstmals wurde in Rio de Janeiro mehr auf digitalen Ausspielwegen der RhB gesendet (243.469 Stunden), als im linearen Fernsehen (113.455 Stunden) (IOC, 2016, S. 4).

\section{Abbildung 2: Broadcast-Hours - verfügbare Sendestunden}

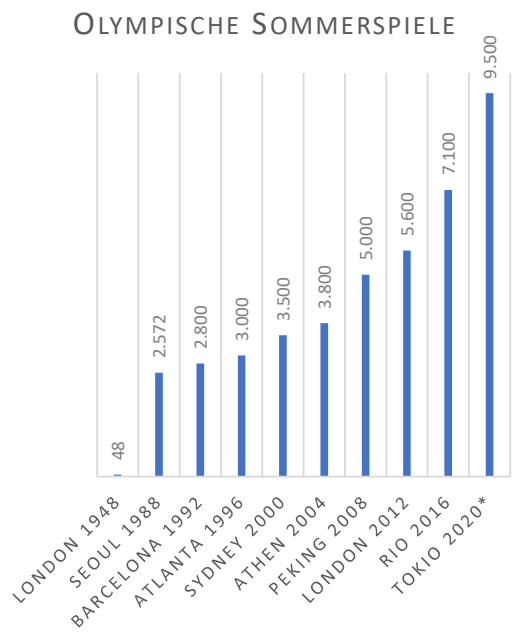

OLYMPISCHE WINTERSPIELE

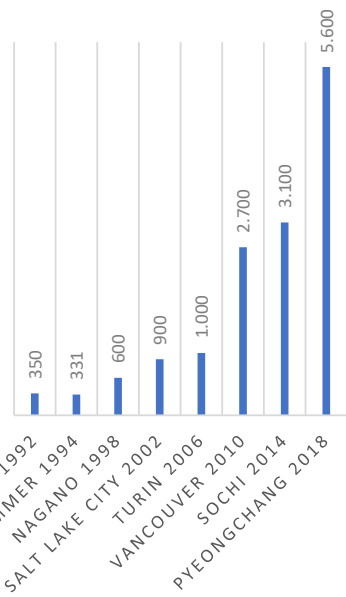

(Hampton, 2008; Mason, 2020; IOC, 2020a, S. 25) 
Abb. 2 zeigt die Anzahl der Stunden, die der Host Broadcaster bzw. OBS den Rechteinhabern in Form von Live-feeds (alle verfügbaren Signale) oder fertig produzierten Inhalten zur Verfügung stellte. Angenommen, die vollumfängliche Berichterstattung hätte in Rio 2016 drei Tage vor Beginn der Spiele begonnen, ein TV-Sender hätte an 20 Sendetagen (17 Wettkampftage) auf 16 Kanälen ein 24-Stunden-Programm anbieten müssen. Tatsächlich wurden von den Olympischen Spiele laut OBS weltweit 350.000 Stunden auf 500 TV-Kanälen und 250 digitalen Ausspielwegen gesendet. Ein Blick in die Details der Zahlen zeigt, wie vielfältig die Angebote inzwischen geworden sind. Von den 7.100 verfügbaren Sendestunden wurden "nur" 2.800+ Stunden als "live-feeds“ angeboten (Mason, 2020, S. 33). In Rio servierte das IOC/OBS exklusives „behind the scenes"-Material, aus dem Olympischen Dorf oder aus der Aufwärmzone im Olympiastadion, wo Journalist"innen nicht hinkommen. Und die Sportler*innen? Aufgrund einer Entscheidung des Bundeskartellamts musste - zumindest für deutsche Athlet*innen - Regel 40 der Olympischen Charta gelockert werden, in der die Teilnahmebedingungen festgeschrieben sind. Dennoch bleiben nur sehr einschränkte Möglichkeiten, während der Olympischen Spielen über Social-Media-Kanäle oder OnlineSeiten zu berichten und sich selbst zu promoten. Das IOC berichtet selbst und gibt exklusive Einblicke in den olympischen Alltag der Athlet"innen. Es ist wie bei einem Bartergeschäft: Das IOC verleiht den „Olympiasieg“ als einen exklusiven und, zumindest für einige Olympioniken, lukrativen „lifetime award“. Dafür liefern die Athlet*innen „the greatest show on earth", wie die BBC ihre Berichterstattung aus Rio 2016 nannte. Die Digitalisierung der Berichterstattung über die neuen Ausspielwege bringt dem IOC zusätzliche Reichweite, birgt aber auch die Gefahr, dass mit zunehmender Nutzung der digitalen Medien, die Quoten im TV nachlassen. Diese waren aber immer die Maßeinheit zur Festlegung der Preise für TV-Rechte. Horky (2018) schreibt, in den USA habe NBC bei der Berichterstattung in PyeongChang 2018 reichlich an Aufmerksamkeit verloren. Auch in der werberelevanten Zielgruppe der 18- bis 49-Jährigen verzeichnete NBC demnach einen Rückgang von rund 17 Prozent. Eine Refinanzierung der TV-Rechte könnte damit in den kommenden Jahren schwieriger werden, wenn sich die Nutzung vom linearen TV in die digitale Welt verschiebt, siehe Abb. 3. 
Abbildung 3: Zuschauer weltweit in Milliarden

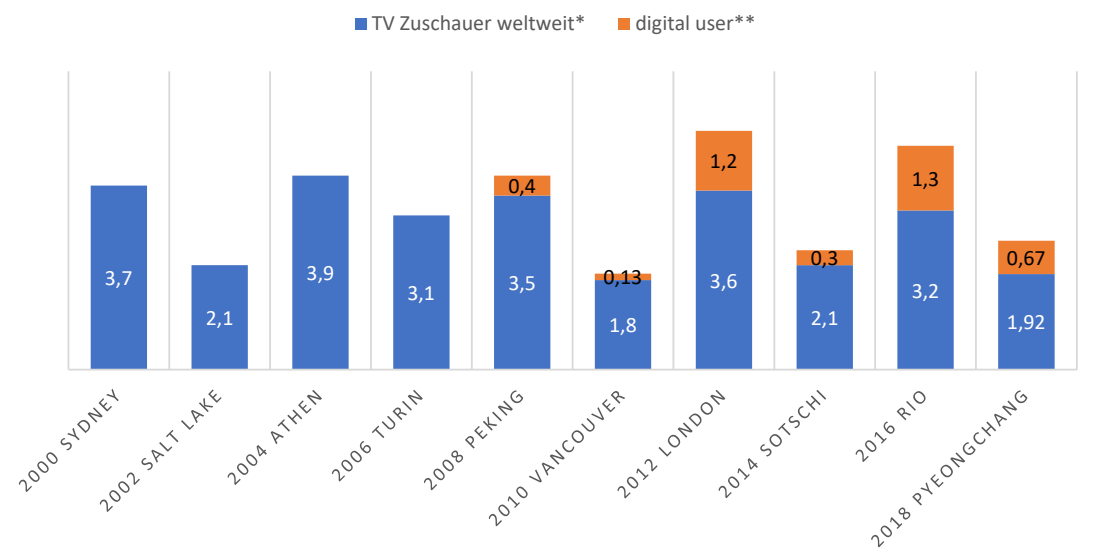

* Anzahl der Zuschauer weltweit, die mindestens eine Minute lang die Berichterstattung über die OS geschaut hat. Das Zahlenmaterial basiert auf den Erhebungen der RhB in den jeweiligen Ländern

*Anzahl der Besucher auf einer Webseite oder einer App der RhB (IOC, 2004a; IOC, 2004b; IOC, 2020a; Mason, 2020)

Für das IOC stellt sich nicht die Frage, ob man die Olympischen Spiele und Werte digital platziert, sondern wie. Eine zentrale Rolle spielt dabei weiterhin der Olympic Channel, mit dem das IOC einen, von großen Plattformanbietern wie Youtube, unabhängigen Sender schuf. Mit Livereportagen und einer Mediathek stellen sie für Olympia-Nerds ein Paradies, für IOC-Partner eine zusätzliche Werbeplattform dar. Die Ansprüche an das IOC/OBS als multimediales Medienunternehmen sind dabei enorm. Wer Sponsoren und TV-Anstalten seine Olympischen Spiele als Premiumprodukt verkauft, der muss auch goldene Gegenleistungen bieten. Deshalb wird auch das IOC nach immer neuen medialen Höchstleitungen streben. Gerade im Zeitalter der Digitalisierung, in dem Produktlebenszyklen keine Olympiade lang halten, ist dies eine tägliche Herausforderung. Das IOC/OBS ist zum Wachsen gezwungen. Dabei „sendet“ der Olympic Channel bereits 365 Tage und die Olympischen Spiele finden eben nur alle vier Jahre statt (im Sommer- und Winterspiele-Wechsel ca. alle zwei Jahre). Was das IOC (1996) kurz vor den Spielen in Atlanta noch futuristisch prophezeite, ist heute gelebte Realität: „die neuen online und multimedia Systeme werden die Art, wie wir das größte Sportereignis der Welt anschauen, für immer verändern“. Und: „Die Spiele werden sich zu einem 
interaktiven Event entwickeln, bei dem Zuschauer nicht mehr nur passive Konsumenten von Informationen sein werden" (IOC, 1996, S. 59). Die Daten dieser Konsument*innen sind das neue Gold. Das „Datensammeln“ wird zu einer Disziplin, in der das IOC künftig einen Medaillenplatz anstrebt. Im Jahr 2019 wurde das „IOC Digital Engagement and Marketing Departement" eingerichtet. Als der neue Direktor Christopher Carroll bei der IOC-Session in Lausanne 2020 die Inhalte der Digitalstrategie vorstellt, penetriert er die IOC-Mitglieder mit dem Begriff "retaining data“ (IOC, 2020b). Daten sammeln oder besser: „Daten behalten und weiterverarbeiten“. Das Ziel der IOC-Digital-Strategie: bis 2026 werden alle Menschen, die sich für olympia-bezogene Inhalte interessieren, über zwei URL bedient: eine URL, die sich an die Fans richtet (www.olympics.com) und eine (www.ioc.org), die die programmatische Arbeit des IOC abbildet (IOC, 2021, S. 19). Die bislang bestehenden Plattformen www.olympic.org und www.olympicchannel.com werden in den neuen URLs verschmolzen. Und wer bekommt die Cookies? Künftig das IOC, das nun endlich seine Kunden und Fans kennt und gezielte Angebote machen kann. Von coolen Filmchen: „... wenn du das magst, könnte dich auch das interessieren“, bis hin zu Merchandising Artikeln und Tickets. Für das Medienunternehmen IOC könnte dabei ein neues Geschäftsfeld entstehen. Dieses Know-How wird das IOC als Servicedienstleistung den Organisationskomitees, den IFs und den NOCs anbieten können. Neue Technologien werden dabei die entscheidende Rolle spielen (Carroll, IOC, 2020b). Das IOC hat sich auch dank der TOP-Sponsoren, die bereits Technologien wie Künstliche Intelligenz (KI), Virtual Reality (VR) oder Augmented Reality (AR) nutzen, einen Wissensvorsprung bei der Organisation der komplexesten Großveranstaltung und bei der „Sportberichterstattung“ auf Multi-TV- und digitalen Plattformen erarbeitet. Das soll die RhB anspornen, die Inhalte für den Sendebetrieb zu perfektionieren. Für die Berichterstattung von den Winterspielen 2018 hatte Eurosport den „CUBE“ entwickelt, ein Studio in Würfelform für digital animiertes VR-/VA-Schulfernsehen. Im Jahr 2019 erhielt EUROSPORT vom IOC den „Golden Rings Award“ für die beste Innovation (IOC, 2019).

Mit dem chinesischen IT-Konzern und IOC-TOP-Partner Alibaba entwickelte OBS ein Cloud-System, das den technischen Aufwand für die Übertragungen erleichtert, Speicherkapazitäten erweitert und RemoteLösungen ermöglicht, mit denen die Berichterstattung von der Heimat aus in Tokio „ferngesteuert“ werden konnte. In ähnlicher Weise wurde die TV-Berichterstattung von ARD und ZDF zur FIFA Fußball-WM 2018 in Russland über einen Sendekomplex beim SWR in Baden-Baden abgewickelt. Das wird auch bei Olympischen Spielen künftig zur Reduzierung 
der Herstellungskosten bei den RhB führen. Computerchip-Hersteller und TOP-Partner Intel entwickelte mit OBS und Alibaba eine datenstabile Pipeline für die noch schnellere Übertragung von Bildern und Daten. OBS (IOC), Alibaba und Intel schaffen neue Synapsen zwischen Athlet*innen und den Medien. So können Athlet*innen beispielsweise 3D-TrackingDaten für VR-Training und Bewegungsoptimierung nutzen. Für TV- und Social-Media-Plattformen der Broadcaster wird das Material grafisch aufbereitet, sendefertig konfektioniert und den Berichterstattern zur Verfügung gestellt. Die internationale Sportwelt dreht sich um das IOC, wird „iocentric“. Alibaba und die OBS-Leute sorgen dafür, dass die Spiele nicht mehr unterbrochen werden: „keeping the Games uninterrupted“ (Alibaba \& OBS, 2021).

\section{Die Rolle der Journalist*innen - Spannungsfeld zwischen Information und Emotion}

Ohne Journalist*innen würden nur die Menschen die Olympischen Spiele kennen, die live vor Ort sind. Journalist*innen berichten, informieren, erklären, vermitteln Nähe, decken Skandale auf. Journalist*innen sind die Vermittler zwischen den Sportler*innen, den Organisator*innen und dem Publikum sowie den Nutzer"innen von Medienangeboten. Neben Sport, Kultur und Umwelt seien die Medien, so referierte Romero (1996), die vierte Säule der Olympischen Bewegung. Bereits in den „Fundamental Principles“, Vorläufer der Olympischen Charta, wurde 1956 festgelegt, dass im Olympiastadion spezielle Plätze zur Verfügung gestellt werden müssen: 1.000 für Journalisten, 150 für Fotografen, 150 für Radio- und Fernsehkommentatoren und Techniker (IOC, 1956b, S. 27). Von den Olympischen Spielen 2008 in Peking berichteten 27.500 Medienvertreter*innen (Mason, 2020, S. 13) über 11.000 Sportler*innen. Politische Einflüsse, Ökonomisierung, nicht zuletzt das Doping haben Haltung und Anforderungsprofil von Medienschaffenden verändert. Neue Technologien der Textverarbeitung, der Bild- und Tonbearbeitung sowie der Übertragungsmöglichkeiten und schließlich die Digitalisierung beeinflussen in einem „work in progress“ das interdisziplinäre Anforderungsprofil der Journalist*innen und schaffen völlig neue Berufsbilder. Thomas Burke (1896), Armin Hary (1960), Usain Bolt (2008, 2012, 2016) sind Olympiasieger in derselben Disziplin. Im Laufe der Zeit haben sich die Laufzeiten geändert, geblieben ist hingegen ihre Leistung: 100 Meter geradeaus, so schnell wie möglich. Die Berichterstattung über den 100- 
Meter-Lauf aber hat sich seit 1896 so sehr verändert, wie die Olympischen Spiele selbst.

In dem Moment, in dem der Sport sich der Unterhaltungsindustrie zuwandte, produzierte auch er seine Stars. Zu den Film- und Popstars kamen die Sportstars. Auf den Zuschauerrängen wurden VIP-Bereiche eingeführt, wobei die "very important persons" immer noch nicht die Athlet"innen sind, sondern die zahlungskräftigen Zuschauenden. Willkommen im „Circus Maximus der Moderne“. So sehr die Kommerzialisierung die Olympischen Spiele finanziell stabilisierte und Athlet"innen weltweit die Möglichkeit eröffnete, ihren Lebensunterhalt zu verdienen, so zerstörerisch sind die kommerziellen Kräfte auch, die auf den gesamten Sport wirken. Die Gier nach Geld und Macht packte auch Funktionär*innen, Athlet*innen und ihre Entourage. Mit dem Dopingfall des Kanadiers Ben Johnson im 100-Meter-Lauf der Spiele in Seoul 1988 und dem öffentlichen Zweifel am „sauberen Image“ der Olympischen Athlet"innen waren Sportjournalist"innen gezwungen, sich mit neuen Disziplinen zu beschäftigen: Medizin, Biochemie, Rechtsfragen. Und Journalist*innen mussten sich immer öfter fragen lassen und sich selbst hinterfragen, ob die "Superstars“, zu denen sie die Sportler"innen schnellschreibend gemacht hatten, vielleicht doch nicht so sauber und heldenhaft sind. Beim Ethik-Seminar des Nationalen Olympischen Komitees 1989 sagte der damalige Sportchef der FAZ, Steffen Haffner (1990, S.61): "Schon heute schwanken Sportjournalisten krass zwischen dem Hochjubeln, dem Heroisieren von Athleten zu Übermenschen und dem lustvollen Niederreißen von Denkmälern." Im Wandel von der Information zur Emotion verringerte sich zwangsläufig die journalistische Distanz zu Sportler“innen. Nach dem Prinzip der „kommunizierenden Röhren“ muss es heute beides geben. Die emotionalen Marktschreier, die den 100-MeterLauf im Stakkato über die Ziellinie brüllen - „telecracy“, wie Lenk (2007, S. 39) schrieb, und die Investigativen, die nach Hinweisen von Whistleblowern den Bestechungsskandal um die Vergabe der Olympischen Winterspiele 2002 an Salt Lake City veröffentlichten oder die den immer noch aktuellen russischen Dopingskandal recherchieren und publizieren. Die pure Sportberichterstattung funktioniert längst nicht mehr. Das IOC ist zwar eine Non-Profit-Organisation, verdient und verteilt aber Milliarden Dollar mit der ökonomischen und medialen Vermarktung von „Olympischen Werten“. Daran muss sich das IOC messen lassen. Der Jubel hat nachgelassen, seit der Zweifel am „sauberen Sieger“ mitläuft. Umweltprojekte von TOP-Partnern, das IOC-Flüchtlingsteam, die Finanzierung von benachteiligten Sportler“innen über „Olympic Solidarity“, das „Educational Programme for Young Reporters“ - in der Reduzierung von Infor- 
mationen auf Schlagzeilen ist dafür selbst in der digitalen Medienwelt kaum Platz. Im Überangebot der Nachrichten gehen die "good news“ unter. Die Nachrichtenquellen sprudeln wie nie zuvor, die Digitalisierung beschleunigt den Informationsfluss und reduziert die Haltbarkeit von Exklusivinformationen. Benötigten Vereine, Verbände, Veranstalter einst die Medien, um ihre Informationen und Botschaften an die Öffentlichkeit zu bringen, sind sie nun selbst Publizisten als „News Producing Companies“. Sie handeln als Informations-Broker, liefern Interviews von Funktionär*innen und Sportler*innen und das ungefiltert, unkritisch. Dabei übertrifft Social Media alles, was man an Kommunikation bisher kannte. Selbst Athlet*innen werden als „Ich-AG“ zu publizistisch Tätigen und Selbstvermarktern. Das Smartphone ist Schreibmaschine und Sendeanstalt in einem. Vorbei sind die Zeiten, in denen Pressemitteilungen an Nachrichtenagenturen geschickt oder einem bekannten Medienschaffenden "gesteckt" wurden. Twitter, Facebook, Instagram \& Co. sind Quellen, über die sich die Medien nun selbst aktiv informieren müssen und aus einem „Post“ oder einem „Tweet“ eine Meldung machen. Mit der Produktion von immer mehr Nachrichten, in immer schnellerem Tempo über immer mehr Plattformen, müssen sich die Medienschaffenden, müssen sich Athlet*innen und vielmehr noch das IOC als „Hüter des Olympismus" allerdings fragen, ob immer mehr auch immer mehr Wert bedeutet. 


\section{Literatur:}

Adi, A. (2013). Media Regulations and the Olympic Charter: a history of visible changes. Journal of Olympic history, 1, 48-57.

Alibaba \& OBS (2021). Intelligent Olympic Games powered by data. Abgerufen am 21.01.2021 unter https://olympics.alibabacloud.com/data-olympics?spm=a2c5o. 217131.1347808.3.2e843a6cW7h9Do

Bach, T. (1994). Paper no. 3210. The Centennial Congress: texts, summaries and plans of papers. IOC. Lausanne.

Bach, T. (2013). Unity in Diversity. Candidature for the Presidency of the International Olympic Committee. Tauberbischofsheim.

Bach, T. (2020). Pressegespräch mit deutschen Journalisten am 12.12.2020. Videokonferenz. Mainz \& Lausanne.

Barney, R., Wenn, S., \& Martyn, S. (2002). Selling the five rings. The International Olympic Committee and the rise of Olympic commercialism. Salt Lake City: The University of Utah Press.

CNNMoney. (2000). NBC to stake Quokka. 22.08.2000. Abgerufen am 20.01.2021 unter https://money.cnn.com/2000/08/22/deals/quokka/

Deutsche Presse Agentur (1993). Olympischer Rekord: EBU zahlte 250 Millionen Dollar für Atlanta. Meldung (ohne Autor). 26.01.1993. DPA: Hamburg

Deutscher Bundestag (1975). Unterrichtung durch die Bundesregierung betr. Gesamtfinanzierung der Olympischen Spiele 1972. Drucksache 7/3066 vom 09.01.1975. Abgerufen am 28.08.2020 unter http://dipbt.bundestag.de/doc/btd/07/030/07030 66.pdf

DOZ (1974). Bericht über die Tätigkeiten der Deutschen Rundfunkanstalten bei den Spielen der XX. Olympiade München 1972 und den Spielen des FIFA Weltpokals. München: Bergverlag Rudolf Rother GmbH.

Downing, T. (1996). A historical perspective. In International Olympic Committee (Hrsg.), The Olympic Movement and the Mass Media. 26-30. Lausanne: IOC.

Haffner, S. (1990). Medien im Sport: Mittler der Moral, Vermittler ohne Moral? „Erst das Siegen, dann die Moral?“. Dokumentation zum Ethik-Seminar des Nationalen Olympischen Komitees für Deutschland. Frankfurt/M.

Hampton, J. (2008). The Austerity Olympics. When the Games came to London in 1948. London: Aurum Press.

Horky, T. (2018). Olympia im US-TV - sinkende Quoten, auch bei jüngeren Zuschauern. Verband Deutscher Sportjournalisten. Abgerufen am 28.02.2018 unter https:/www.sportjournalist.de/VDS-Nachrichten/Meldungen/;2711-Olympia_ im_US-TV

IOC (1956a). Minutes of the meeting of the Executive Board of the I.O.C., held in Lausanne. 3.- 4.10.1956. Lausanne.

IOC (1956b). The Olympic Games. Fundamental Principles, Rules and Regulations. General Information. Abgerufen am 29.11.2020 unter https://ibrary.olympic.org/ Default/doc/SYRACUSE/61937/the-olympic-games-fundamental-principles-rules -and-regulations-general-information-international-oly 
IOC (1958). The Olympic Games. Fundamental Principles, Rules and Regulations. General Information. Abgerufen am 29.11.2020 unter https://ibrary.olympic.org/ Default/doc/SYRACUSE/61944/the-olympic-games-fundamental-principles-rules -and-regulations-general-information-international-oly

IOC (1996). The Olympic Games and the media. Feature by the International Telecommunications Union (ITU). Olympic Review, XXV (9). 57-63. Lausanne: IOC.

IOC (2004a). IOC Marketing Report Athens 2004. 77-89. Abgerufen am 22.11.2020 unter https://library.olympic.org/Default/doc/SYRACUSE/64957/athina-2004marketing-report-international-olympic-committee

IOC (2004b). Athens 2004 Olympic Games Global television Report. Abgerufen am 06.01.2021 unter https://stillmed.olympic.org/Documents/Reports/EN/en_ report_1086.pdf

IOC (2014a). IOC awards Olympic Broadcast Rights to NBCUniversal through 2032. 07.05.2014. Abgerufen am 02.10.2020 unter https://www.olympic.org/news/ioc -awards-olympic-games-broadcast-rights-to-nbcuniversal-through-to-2032

IOC (2014b). Marketing Commission - fonds list. August 2014. Historical Archives. Abgerufen am 06.01.2021 unter https://stillmed.olympic.org/AssetsDocs/OSC\% 20Section/pdf/LRes_Fonds_list_marketing_commission.pdf

IOC (2014c). Olympic Agenda 2020 - Context and Background. Lausanne: IOC

IOC (2016). Global Broadcast and Audience Report. Olympic Games Rio 2016. Abgerufen am 06.01.2021 unter https://library.olympic.org/Default/doc/ SYRACUSE/166292/global-broadcast-and-audience-report-olympic-games-rio -2016-international-olympic-committee?_lg=en-GBIOC

IOC (2018). Alibaba and OBS collaborate for Tokyo 2020 Broadcasting Cloud Solution. 21.09.2018. Abgerufen am 01.01.2021 unter www.olympic.org/news/alibaba-and -obs-collaborate-for-tokyo-2020-broadcasting-cloud-solution

IOC (2019a). Consolidated Financial Statements. 116-171. In: The IOC Annual Report 2019. Abgerufen am 10.10.2020 unter: https://stillmedab.olympic.org /media/Document\%20Library/OlympicOrg/Documents/IOC-Annual-Report/ IOC-ANNUAL-REPORT-2019.pdf\#_ga=2.40693157.620999333.1611492137-743 783901.1604740129

IOC (2019b). Olympic Golden Rings Award winners unveiled in front of 1,000 guests in Lausanne. IOC News (22 Jun 2019). Abgerufen am 12.12.2020 unter https:/www.olympic.org/news/olympic-golden-rings-award-winners-unveiled-in -front-of-1-000-guests-in-lausanne

IOC (2020a). Olympic Marketing Fact File. Updated January 2020. Abgerufen am 23.11.2020 unter https://stillmedab.olympic.org/media/Document\%20Library/ OlympicOrg/Documents/IOC-Marketing-and-Broadcasting-General-Files/ Olympic-Marketing-Fact-File.pdf\#_ga=2.247269379.620999333.1611492137743783901.1604740129

IOC (2020b). 135th IOC Session. Youtube Kanal des IOC. Abgerufen am 24.02.2020 unter https://www.youtube.com/watch?v=rihy7JLpTwo 
IOC (2021). Olympic Agenda 2020 + 5. 15 recommendations. Abgerufen am 05.04.2021 unter https://www.olympic.org/olympic-agenda-2020-plus-5

Killanin, M. (1983). My Olympic Years. New York: Morrow.

Lenk, H. (2007). An Anthropology of the Olympic Athlete Towards a Modernised Philosophy of the Olympic Games and Athletes. Journal of Olympic History, 1, 39-47.

Llinés, M., \& Moreo, A. (1998). The History of Radio and Television coverage of the Olympic Games. Television in the Olympic Games the New Era. Lausanne: IOC.

Lyberg, W. (2004). Book Reviews - Samaranch, J.A., Memorias Olímpicas. Journal of Olympic History, 12, 48.

Maney, K. (2000). Quokka competes for Olympic glory. USA Today. 25.02.2000

Mason, M. (2020). OBS Olympic Broadcasting History 2001 - 2018. Unveröffentlichte Dokumentation. Madrid: OBS.

Michel, R. (1972). Erfahrungsbericht Olympische Spiele München und Kiel 1972. Privatarchiv.

Moragas, Miquel de (1999): Olympic Movement and Information Society: new Internet challenges and opportunities. Barcelona: Centre d'Estudis Olímpics UAB. Abgerufen am 02.10.2020 unter http://olympicstudies.uab.es/pdf/ wp101_eng.pdf

Pound, R. (1999). Olympic Marketing Fact File Winter 1999. Lausanne. IOC

Pound, R. (2004). Inside the Olympics. A behind-the-scenes look at the politics, the scandals, and the glory of the Games. Toronto/Etobikoke: Wiley.

Reichs-Rundfunk (1936). Olympia-Weltsender. Berlin

Romero, M., \& Gavilan, E. (1993). HDTV Coverage of the Barcelona Olympic Games. EBU Technical review Winter 1992. Abgerufen am 12.12.2020 unter www.tech.ebu.ch

Simon, M. (2000). Olympia 2000: Das Netz als Sieger mit Handicap. Teleopolis. Abgerufen am 04.01.2021 unter https:/www.heise.de/tp/features/Olympia-2000Das-Netz-als-Sieger-mit-Handikap-3451055.html

SPIEGEL (1972). Olympia - das totale Fernsehen. 28. August 1972. 36. 24-38. Hamburg. 
